\title{
Spatial double physical pendulum with axial excitation: computer simulation and experimental set-up
}

\author{
Michał Ludwicki • Jan Awrejcewicz • Grzegorz Kudra
}

Received: 15 January 2014 / Revised: 6 February 2014 / Accepted: 27 February 2014 / Published online: 28 March 2014

(C) The Author(s) 2014. This article is published with open access at Springerlink.com

\begin{abstract}
Physical and mathematical model of a threedimensional double physical pendulum being coupled by two universal joints is studied. Upper joint of this pendulum is periodically rotated in its axial direction. Damping forces and torques inside joints as well as an influence of the gravitational field and damping forces and torques inside joints are taken into account during derivation of the ODEs of this mechanical system. The work consists of mathematical modelling, computer simulation and parts of the experimental analysis to confirm the numerical simulations. The experimental setup realizes kinetic excitation by an nonconstant periodic torque of servomotor, controlled by the computer. Four incremental encoders are mounted on pendulum joints, so the angles of rotation of the pendulum are measured in real-time and analysed by an originally developed acquisition software. Future plans of the mathematical and experimental model are also discussed. Presented exemplary results showed a number of non-linear effects, including chaos, quasi-periodic and periodic dynamics.
\end{abstract}

Keywords Double spatial physical pendulum - Chaotic dynamics · Universal joint · Computer simulation

\footnotetext{
M. Ludwicki $(\bowtie) \cdot J$. Awrejcewicz · G. Kudra

Department of Automation, Biomechanics and Mechatronics, Lodz University of Technology, Lodz, Poland

e-mail: michal.ludwicki@p.lodz.pl

J. Awrejcewicz

e-mail: jan.awrejcewicz@p.lodz.pl

G. Kudra

e-mail: grzegorz.kudra@p.lodz.pl
}

\section{Introduction}

We consider the mathematical model of a simple threedimensional (3D) double physical pendulums system, under specific conditions. The mechanism is physically excited by a periodic torque in axial direction and linearly damped by each joint. This excitation is applied to the suspension joint of the mechanism. Such an approach (damping and excitation) is often used in many pendulum analysis but rarely the excitation has axial direction.

There are many examples of simple mechanical systems that exhibit complex behavior under certain conditions. Common pendulum, e.g. a clock by Huygens [1], under typical situations, are not enough interesting in term of nonlinear dynamics. It appears here that the conditions are more important than the mechanisms itself. The pendulum became highly fascinating under specific circumstances, e.g. influence of excitation and damping.

The evolution of pendulum analysis started from the measurements and experiments, e.g. famous Foucault's pendulum, 1851 [2,3] or Kater's reversible pendulum [4]. Nowadays pendulum mechanisms are more often used in theoretical or practical mathematical modeling process, for example, to develop more effective vibrations absorption methods $[5,6]$. There are also advanced research about the pendulum itself, including its experimental identification $[7,8]$ or control algorithms [9].

Multiple pendulum systems are mostly simplified either to planar vibrations $[10,11]$ or they concern only mathematical pendulums [12]. Physical pendulums are examined in their multiple configuration, e.g. $[13,14]$ but also in some simplified forms.

This paper presents the results of numerical computations, as well as the possibilities of applications of the original simulation program. A wide range of nonlinear behavior of the 


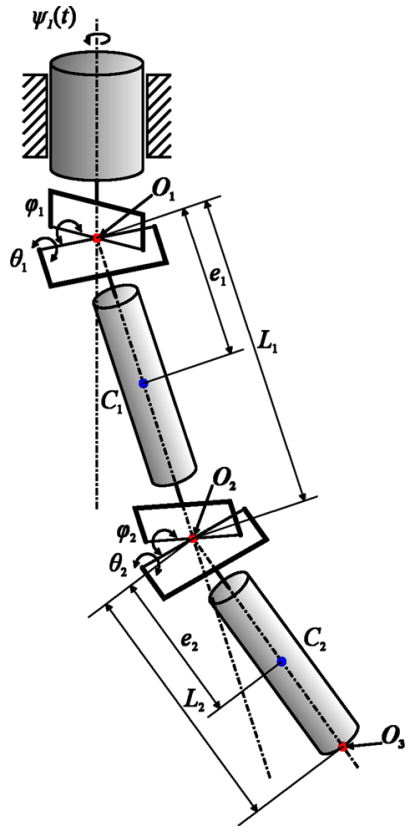

Fig. 1 Coupled pendulums

system is noticed. Additionally, some results of simulations for the ready built experimental setup are presented.

\subsection{The Pendulum Model}

The system to be considered here is shown in Fig. 1. It is build of two cylindrical-shaped rigid bodies combined by universal joint $O_{2}$ and hung on a second universal joint $O_{1}$. This joint is externally driven and it actuates the entire mechanical system axially with either constant or non-constant angular velocity. Influence of the gravitational force is also included. The only damping force that the model contains is inside joints and it is characterized by a simple viscous damping function. Air resistance is neglected due to low velocities of vibrations and relatively large moments of inertia.

Angular positions of each universal joint's shaft have been described by three Euler angles $\varphi_{i}, \theta_{i}$ and $\psi_{i}$, where $i$ corresponds to an index of each joint. The rotation matrices are found, as well as positions of each body centers. Also its linear and angular velocities and energy are defined.

Analytically determined set of nonlinear ODEs governing the pendulum dynamics follows

$$
\begin{aligned}
& \mathbf{M}(\mathbf{q}) \ddot{\mathbf{q}}+\mathbf{A}(\mathbf{q}) \mathbf{a}+(\omega(t) \mathbf{B}(\mathbf{q})+\mathbf{C}) \dot{\mathbf{q}}+\mathbf{r}_{g}(\mathbf{q}) \\
& \quad+\omega^{2}(t) \mathbf{r}_{\omega}(\mathbf{q})=\mathbf{0}
\end{aligned}
$$

where $\mathbf{q}=\left[\theta_{1}, \varphi_{1}, \theta_{2}, \varphi_{2}\right]^{T}, \mathbf{a}=\left[\dot{\theta}_{1}^{2}, \dot{\varphi}_{1}^{2}, \dot{\theta}_{2}^{2}, \dot{\varphi}_{2}^{2}, \dot{\theta}_{1} \dot{\varphi}_{1}\right.$, $\left.\dot{\theta}_{1} \dot{\theta}_{2}, \dot{\theta}_{1} \dot{\varphi}_{2}, \dot{\theta}_{2} \dot{\varphi}_{1}, \dot{\varphi}_{1} \dot{\varphi}_{2}, \dot{\theta}_{2} \dot{\varphi}_{2}\right]^{T}$, and $\mathbf{M}, \mathbf{A}, \mathbf{B}, \mathbf{C}, \mathbf{r}_{g}, \mathbf{r}_{\omega}$ devote matrices and vectors (here not defined explicitly).
Analytical Wolfram Mathematica ${ }^{\circledR}$ computer package has been carried out, during process of derivation of Eq. (1). Full form results are too large and couldn't be simplified enough to show in this paper.

In the study, simple model of viscous damping of joints is assumed in the following form:

$\mathbf{M}_{d}=\left[\begin{array}{llll}M_{d \theta_{1}}-M_{d \theta_{2}}, & M_{d \varphi_{1}}-M_{d \varphi_{2}}, M_{d \theta_{2}}, M_{d \varphi_{2}}\end{array}\right]^{T}$,

where $\mathbf{M}_{i}$ are corresponding damping torques proportional to the angular velocities.

Angular velocity of the axial excitation of first joint follows:

$\dot{\psi}_{1}=\omega(t)=\omega_{0}+q \sin (\Omega t)$,

where $\omega_{0}$ is a constant part of velocity ( $\left.\mathrm{rad} / \mathrm{s}\right), q$ is the amplitude $(\mathrm{Nm})$ and $\Omega$ states for frequency $(\mathrm{rad} / \mathrm{s})$.

Unlike experimental setup, the mathematical model does not take into account impacts caused by the mechanical limits of real double spatial pendulum rotation yet. That is why the full comparison of the theoretical model and experimental measurements can be performed for low amplitudes only, smaller than about $90^{\circ}$. However, this does not prevent to achieve some really interesting simulations results based on the experimental setup parameters (not measurement), without angle limits. Full experimental analysis will be performed after including impacts model to the equations of the motion, increase damping coefficients in the experimental joints to prevent such impact and/or preparing the setup to hold out frequent impacts without the risk of failure.

\section{Numerical computations}

Results presented in this paper concern the following fixed parameters (see Fig. 1) presented in Table 1. Values on the left are theoretical ones, chosen for the purpose of exemplary simulations. Two columns on the right presents simulation parameters that corresponds to the measured values of built experimental setup.

According to the User manual of Wolfram Mathematica ${ }^{\circledR}$ package, its ODEs solving algorithm is based on higher order Runge-Kutta methods with automatic step control. Results, as well as the plots, are automatically interpolated to any chosen time steps.

Analytically determined ODEs set (1) was simplified and solved numerically for parameters values showed in Table 1. Every first 500 time steps of all numerical computations 
Table 1 Numerical computation parameters

\begin{tabular}{llllll}
\hline & \multicolumn{2}{l}{ Simulation example } & & \multicolumn{2}{l}{ Experiment } \\
\cline { 2 - 3 } & First joint & Second joint & & First joint & Second joint \\
\hline Weight of the pendulums $(\mathrm{kg})$ & $m_{1}=0.5$ & $m_{2}=0.5$ & & $m_{1}=3.87$ & $m_{2}=2.12$ \\
Length (m) & $L_{1}=0.2$ & $L_{2}=0.2$ & & $L_{1}=0.22$ & $L_{2}=0.2$ \\
Position of the mass center (m) & $e_{1}=0.1$ & $e_{2}=0.1$ & & $e_{1}=0.11$ & $e_{2}=0.039$ \\
Moments of inertia $(\mathrm{kg} \mathrm{m})$ & $I_{x 1}=0.002$ & $I_{x 2}=0.002$ & & $I_{x 1}=0.032$ & $I_{x 2}=0.004$ \\
& $I_{y 1}=0.002$ & $I_{y 2}=0.002$ & & $I_{y 1}=0.025$ & $I_{y 2}=0.007$ \\
& $I_{z 1}=0.0001$ & $I_{z 2}=0.0001$ & & $I_{z 1}=0.009$ & $I_{z 2}=0.005$ \\
Viscous damping coefficient $(\mathrm{N} \mathrm{s} / \mathrm{m})$ & $c_{1}=0.1$ & $c_{2}=0.1$ & & $c_{1}=0.1$ & $c_{2}=0.1$ \\
\hline
\end{tabular}

(a)

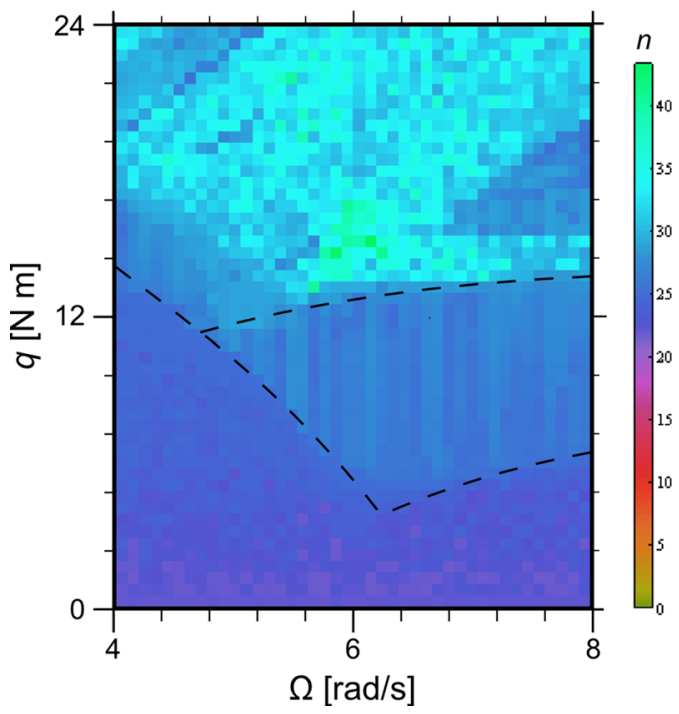

(b)

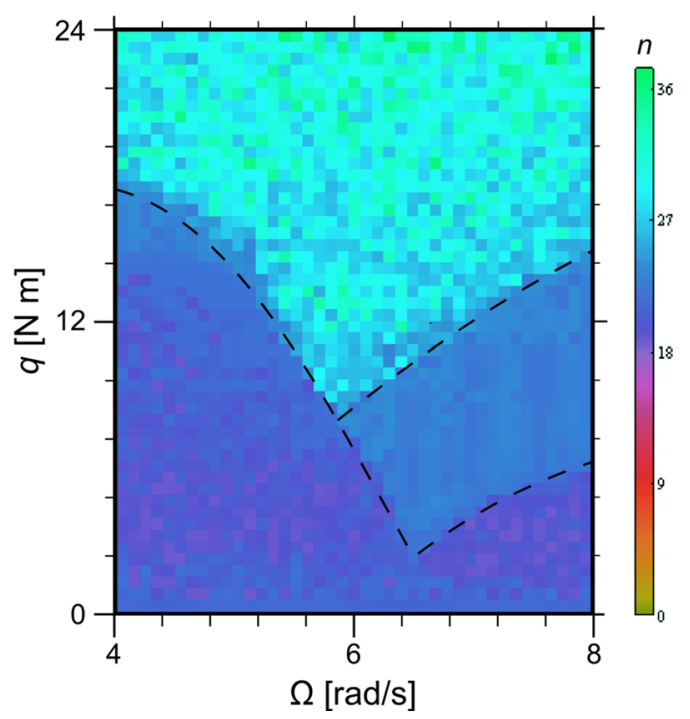

Fig. 2 Number of full $360^{\circ}$ rotations map for angle $\varphi_{1}$ and $\omega_{0}=0$; a exemplary simulation, and $\mathbf{b}$ simulation of built experimental setup

were ignored as transient motion and next 500 or more if needed were qualified as significant for the analysis.

\subsection{Results and analysis}

To find globally how much these two systems (example and experiment, see Table 1) are different, 2D maps of maximum numbers of full $360^{\circ}$ rotations were computed (Fig. 2) for the same range of control parameters $q$ and $\Omega$, while $\omega_{0}$ equals $0 \mathrm{rad} / \mathrm{s}$ (pure periodic excitation).

One can see some similarities between them the maps, especially in the shape of the regions where the number of full rotations are low. These maps need a very long time of computing (here about $31 \mathrm{~h}$ ) but it reveals some general information about the system itself, faster than the bifurcation diagrams.

Using the classical methods of analysis of dynamic systems, the bifurcation diagrams, Poincaré maps and phase plots were produced numerically. In Fig. 3, three nonlinear phenomena are presented. Amplitude of excitation $q$ was set to $12 \mathrm{Nm}$ to cover all three regions of different value of full rotations (see Fig. 2).

While changing value of the control parameter $\Omega$, several types of nonlinear behaviour can be observed, including simple periodic vibrations, e.g. for $\Omega=5.7 \mathrm{rad} / \mathrm{s}$ (see Fig. 3c), quasi-periodic vibrations, e.g. $\Omega=5.5 \mathrm{rad} / \mathrm{s}$ (see Fig. 3a) or wide window of chaotic movement for $\Omega$ around $5.49 \mathrm{rad} / \mathrm{s}$ (see Fig. 3b).

Similar analysis were performed for the parameters that corresponds to built experimental setup. This time, the system also showed a number of interesting non-linear phenomena, reported in Fig. 4. During the simulation process, it appeared that the system is less sensitive to exhibit nonlinear phenomena than the exemplary one. The reason for this is probably a large moments of inertia of the experimental pendulum, and a small damping of motion. Apart from this, it can be seen here also periodic, quasi-periodic and chaotic behaviour of our investigated system. 
(a)
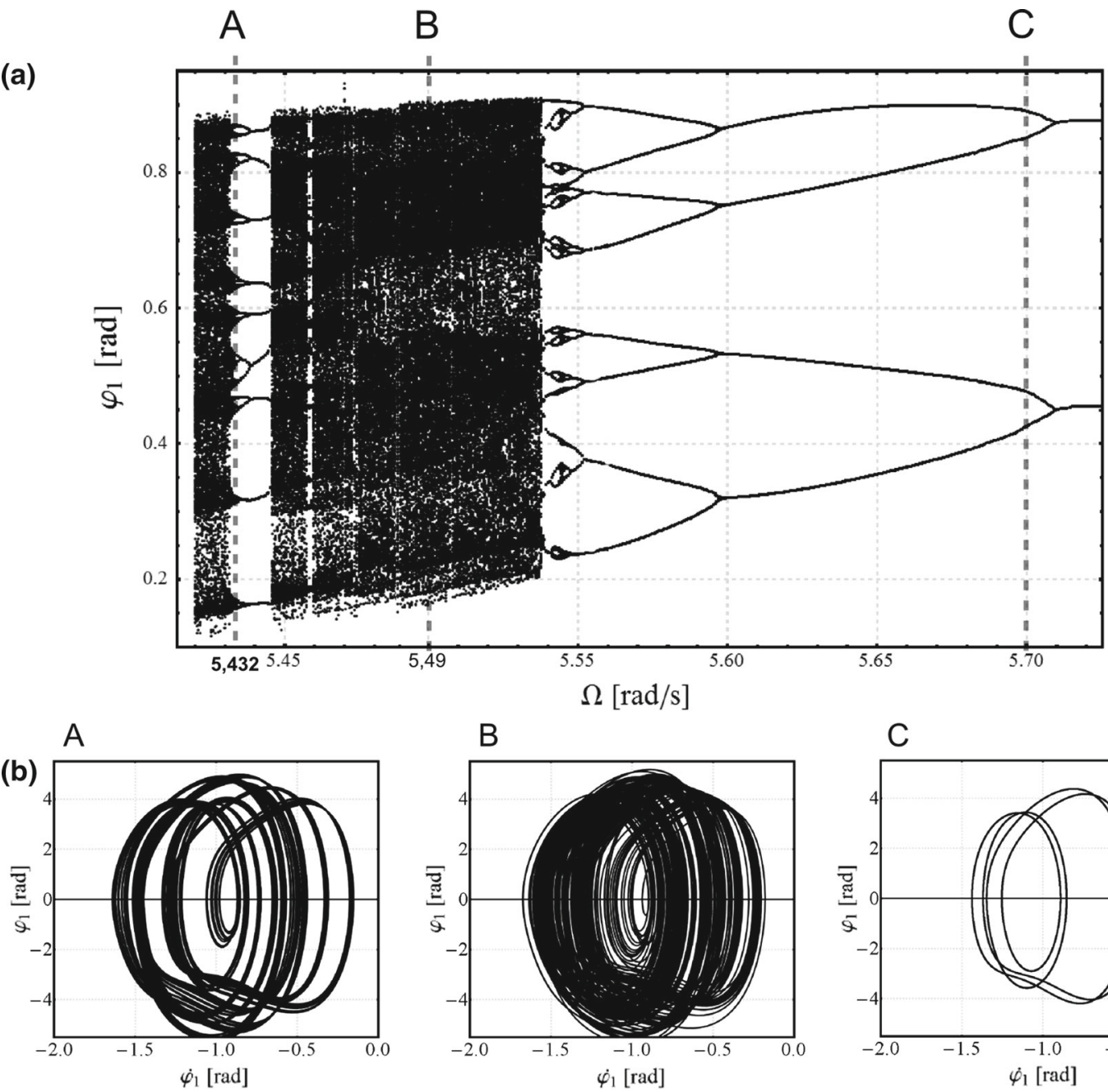

\section{B}
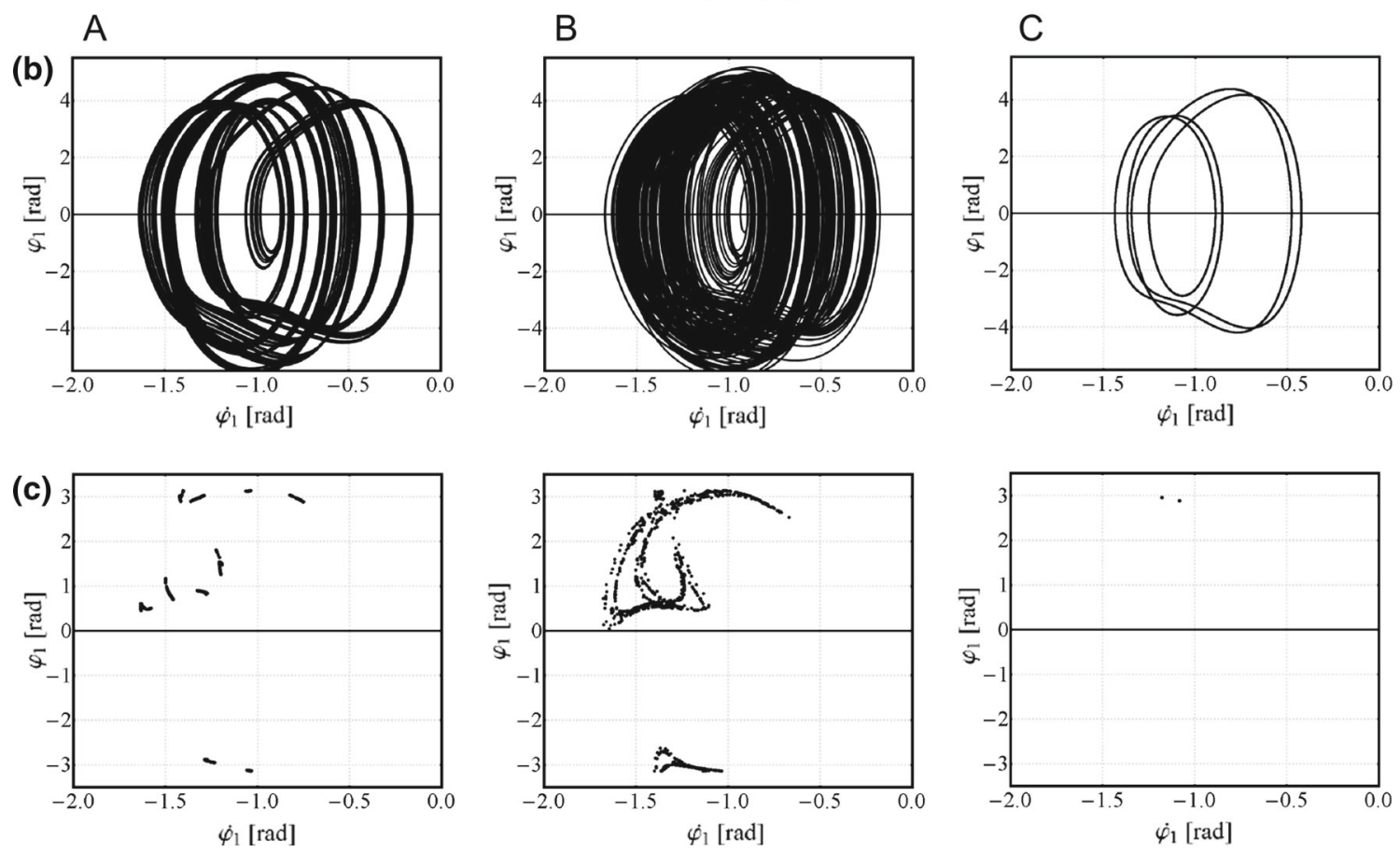

Fig. 3 Bifurcational diagram regarding angle $\varphi_{1}$ in range $\Omega \in\langle 5.42,5.72\rangle \mathrm{rad} / \mathrm{s}$ with step $0.0006 \mathrm{rad} / \mathrm{s}$ for $\omega_{0}=0 \mathrm{rad} / \mathrm{s}$ and $q=12 \mathrm{~N} \mathrm{~m}(\mathbf{a})$, three sets of phase trajectories (b) and Poincaré maps (c) corresponding to three different nonlinear phenomena (for exemplary system parameters)

\section{Experimental rig}

In Fig. 5 one can see the photography and construction scheme of the experimental rig. The original control and acquisition software has been developed, using Java programming language. It can perform in real-time, parallel measurements of values of all four angles of rotation simultaneously. The program can control the parameters of the external excitation and at the same time record each pendulums link position.

The orientation of each pendulum link is measured by four precise incremental encoders and the dedicated PC acquisition card. To transmit signals between rotating pendulums equipped with the encoders and mounting frame, without the risk of the wiring damage, special slip ring is used. It consists of motor-like brushes, which provides a continuous electrical 
(a)
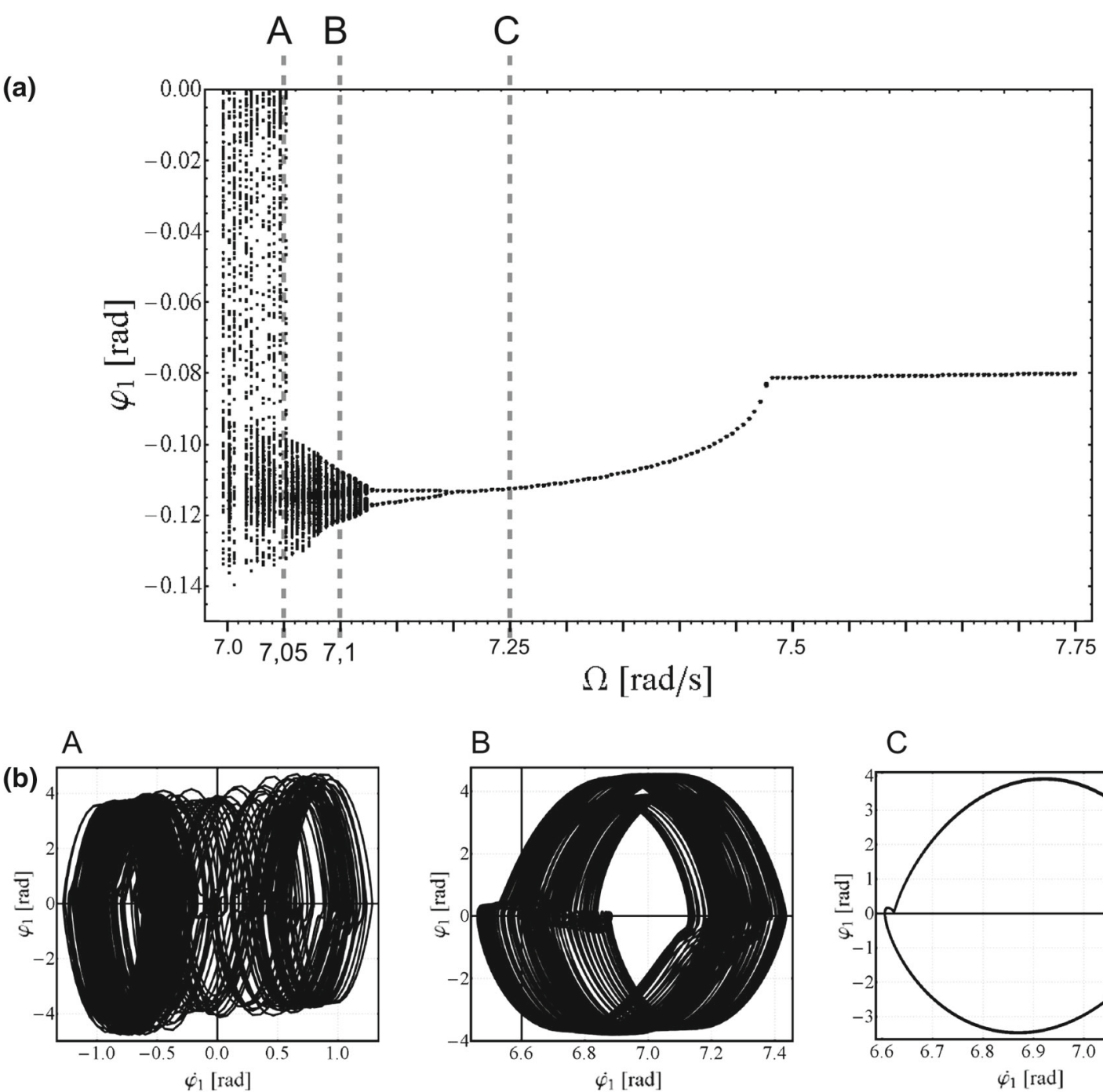

B
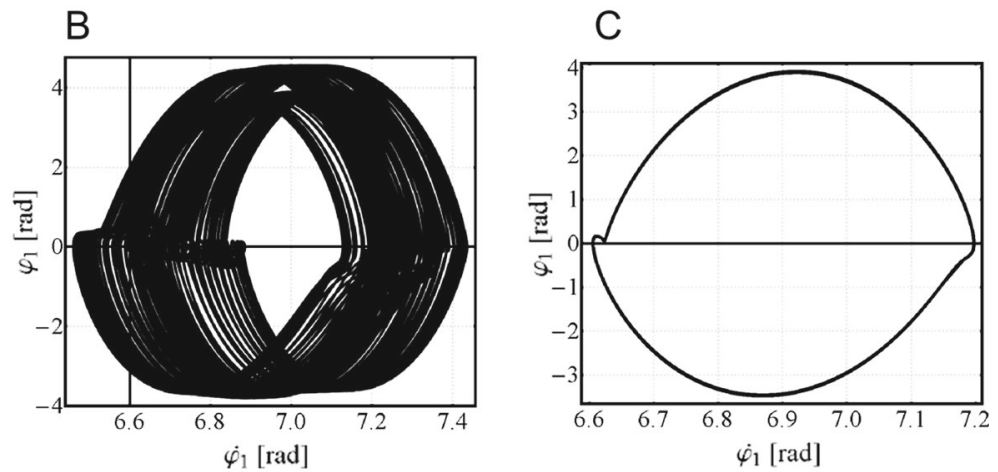

(c)
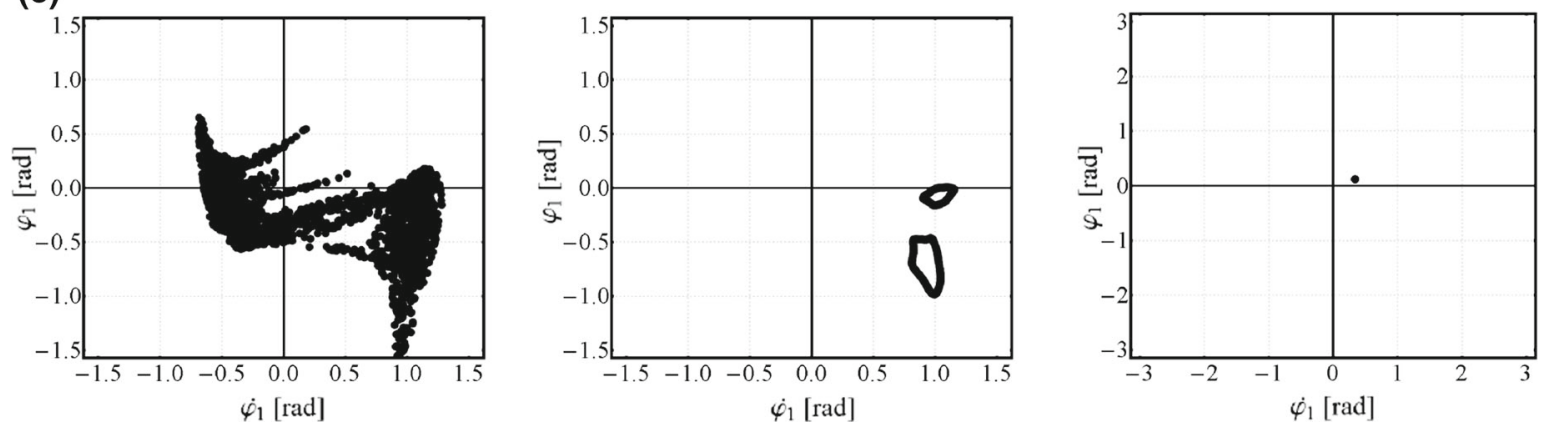

Fig. 4 Bifurcational diagram regarding angle $\varphi_{1}$ in range $\Omega \in\langle 7,7.75\rangle \mathrm{rad} / \mathrm{s}$ with step $0.05 \mathrm{rad} / \mathrm{s}$ for $\omega_{0}=0 \mathrm{rad} / \mathrm{s}$ and $q=12 \mathrm{~N} \mathrm{~m}(\mathbf{a})$, three sets of phase trajectories (b) and Poincaré maps (c) corresponding three different nonlinear phenomena (for experimental setup system parameters)

connection both of measurement signals and of power supplied to the encoders. The external angular velocity excitation is provided by the PC-controlled servomotor.

Some preliminary measurements have been performed. Figure 6 shows a time series measurement under constant angular velocity of excitation equals $3.7 \mathrm{rad} / \mathrm{s}$.
Presented plot of angular positions of each link shows that as the higher frequency vibrations disappears, all four links oscillate simultaneously. Afterwards, because of the influence of the constant axial excitation, the amplitude of vibration increases gradually to reach its constructional limits, and takes place an impact. This impact sce- 

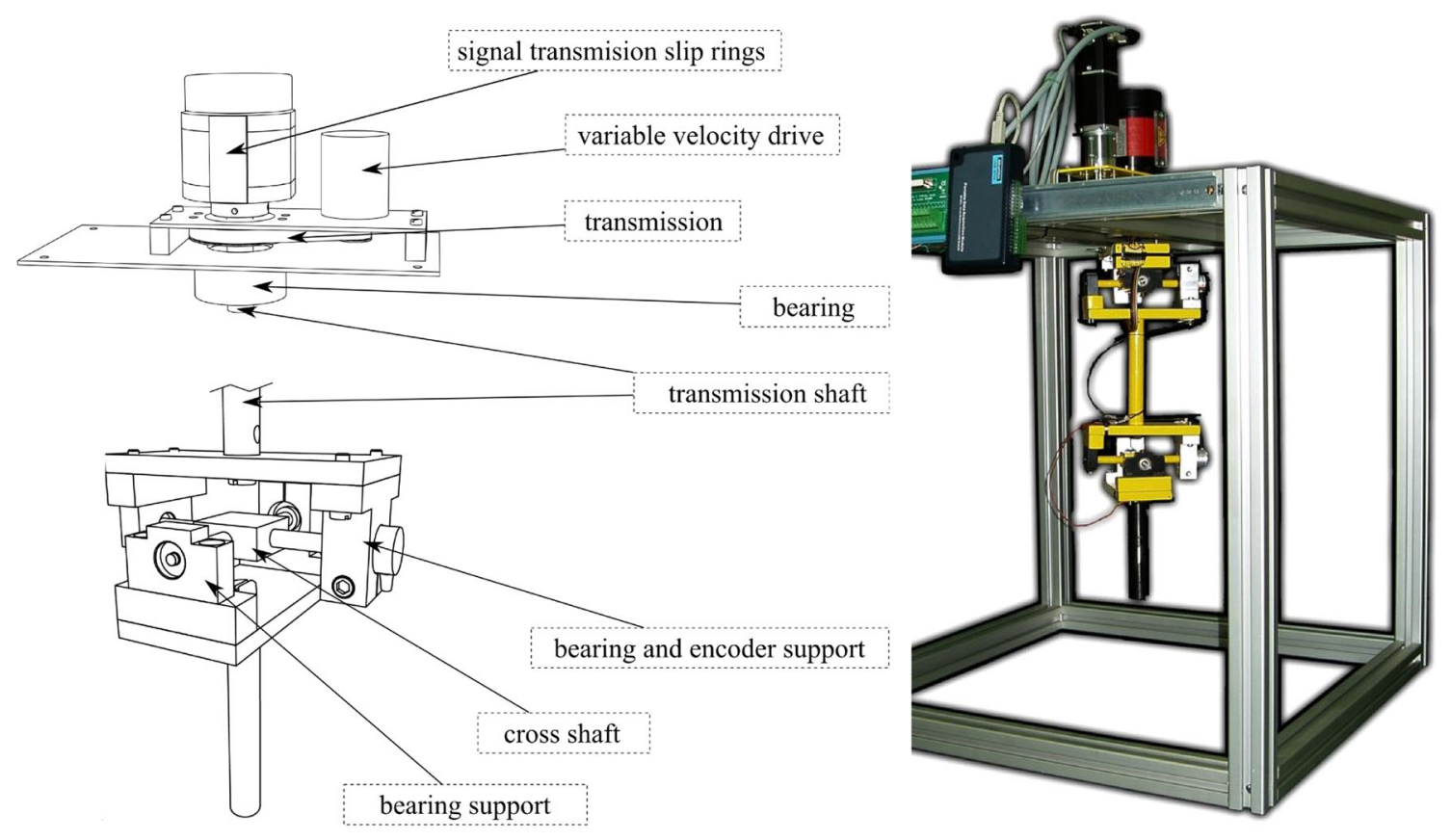

Fig. 5 A part of the designed experimental rig

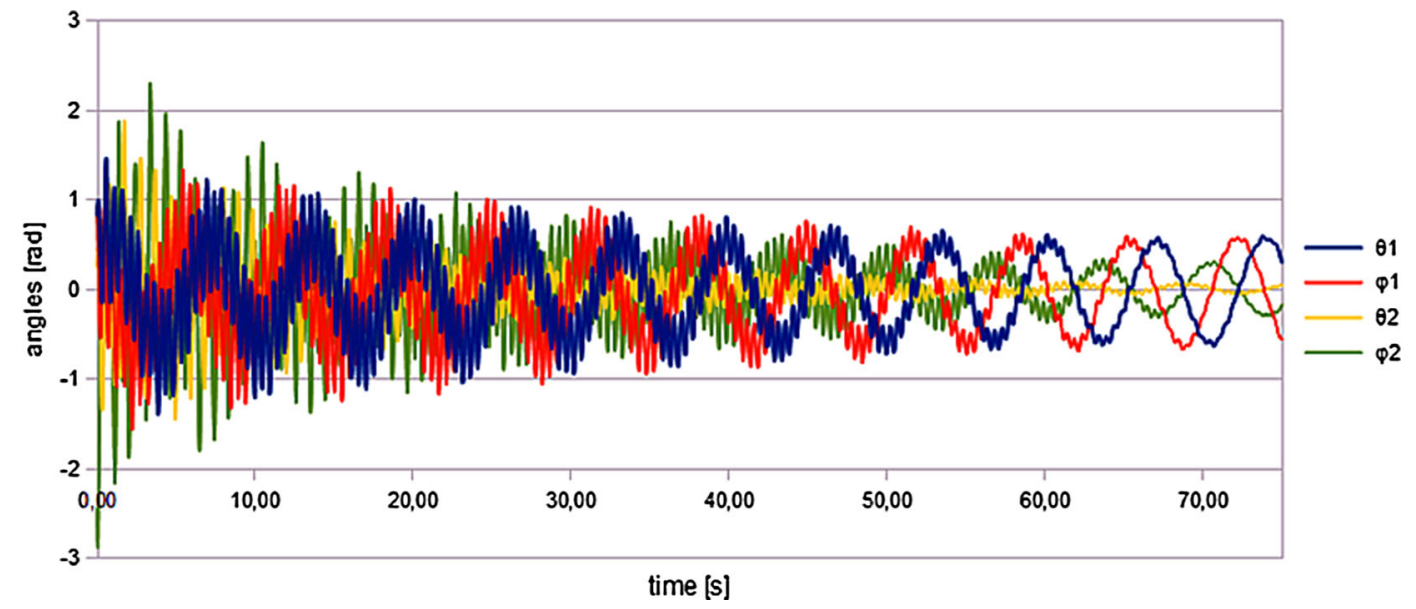

Fig. 6 Time series of angular positions of all two links measured under constant angular velocity of excitation $\omega_{0}=3.7 \mathrm{rad} / \mathrm{s}$, before impacts

nario is presented in Fig. 7. However, the build experimental setup is not prepared for frequent collisions of its links and has to be modified to prevent its damage.

As showed in Fig. 8, the measurement equipment is enough fast to make some draft phase plots. It should be noted that there is no direct information about the angular velocities of the links, and they have to be calculated numerically. Applied numerical differentiation algorithm makes the plot quite discrete but sufficiently suitable for further generation of bifurcation diagrams or other data analysis.

\section{Conclusions}

As it was presented before, double physical pendulum with axial excitation, either in exemplary and experimental configuration, exhibits a wide spectrum of nonlinear effects. Periodic, quasi-periodic and chaotic orbits have been found and discussed, among others. Preliminary measurement data plots have been also showed and shortly discussed. Numerically generated full $360^{\circ}$ rotation maps of the pendulum links show that both configurations reveals some similarity, despite of the fact that its geometrical and mechanical parameters differ significantly. 
Fig. 7 Time series of angular positions of all two links measured under constant angular velocity of excitation $\omega_{0}=3.7 \mathrm{rad} / \mathrm{s}$, ended with impacts

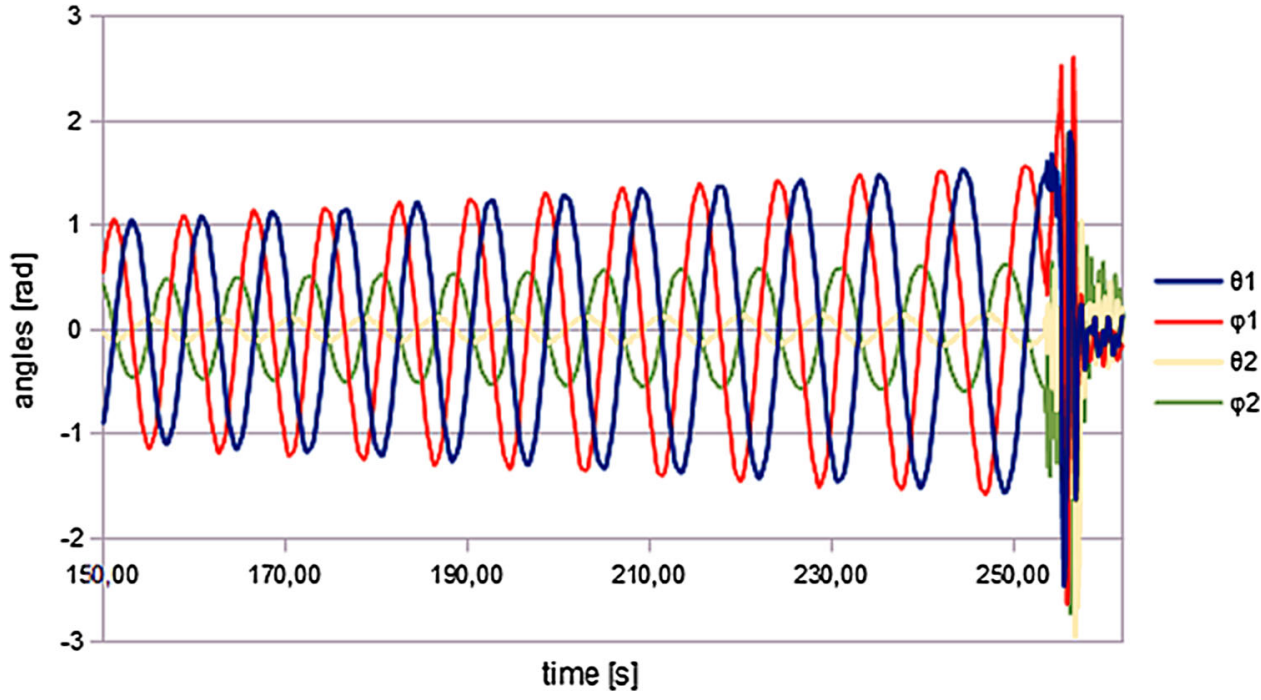

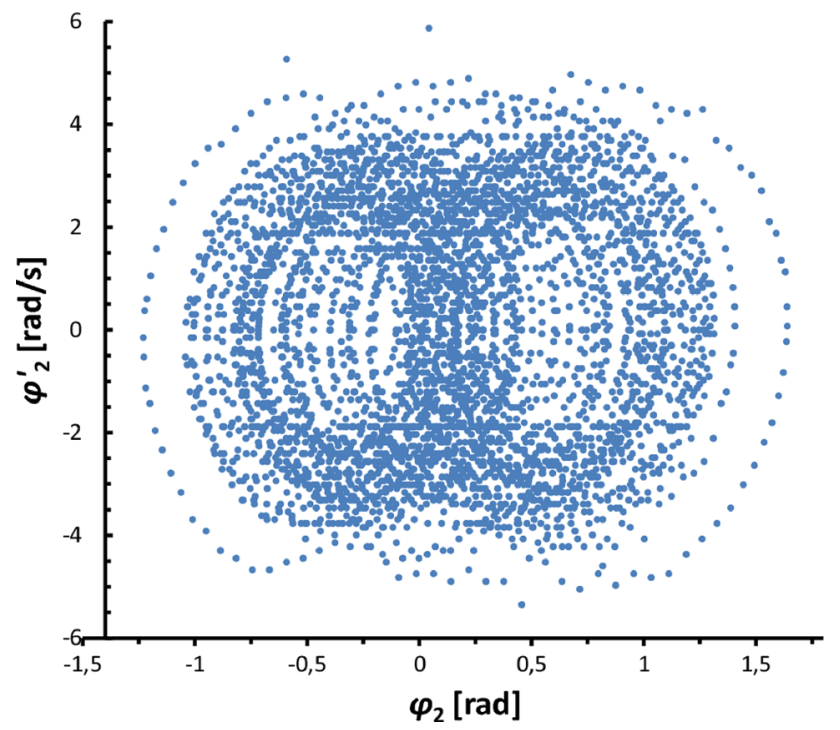

Fig. 8 Phase trajectories of the second link measured under constant angular velocity of excitation $\omega_{0} \approx 3.7 \mathrm{rad} / \mathrm{s}$, for low amplitudes, before impacts

Presented figures and the carried out study confirm that performed analytical and numerical calculations can be used to analyse and simulate multiple pendulum systems and other similar multiple rigid body mechanical systems.

The next step of this project is to extend the mathematical model to include impacts. Additionally, the experimental setup has to be upgraded, to make possibility of modifying the damping coefficient of all pendulums links (ball bearings) and to automatically detect all potential impacts occurrence. Rapid responsible impacts detection is necessary to make a long-time measurements guiding to Poincaré maps and bifurcation diagrams generation. That will make possibility to perform full identifi- cation of built system with no other mechanical limitations.

Acknowledgments This work has been originally presented on International Conference-12th Dynamical Systems-Theory and Applications 2013. This paper was financially supported by the National Science Centre of Poland under the Grant MAESTRO 2, number 2012/04/A/ST8/00738, for years 2013-2016.

Open Access This article is distributed under the terms of the Creative Commons Attribution License which permits any use, distribution, and reproduction in any medium, provided the original author(s) and the source are credited.

\section{References}

1. Yoder JG (2005) Chapter 3. In: Huygens C (ed) Book on the pendulum clock (1673) - landmark writings in Western mathematics 1640-1940. Elsevier Science, Amsterdam, pp 33-45

2. Condurache D, Martinusi V (2008) Foucault Pendulum-like problems: a tensorial approach. Int J Nonlinear Mech 43(8):743-760

3. Aczel AD (2003) Pendulum: Leon Foucault and the triumph of science. 1st Atria. Atria, New York

4. Kater H (1818) An account of experiments for determining the length of the pendulum vibrating seconds in the latitude of London. Philos Trans R Soc Lond 108:33-102

5. Matta E, De Stefano A (2009) Seismic performance of pendulum and translational roof-garden TMDs. Mech Syst Signal Process 23(3):908-921

6. Shang-Teh W (2009) Active pendulum vibration absorbers with a spinning support. J Sound Vib 323(1-2):1-16

7. Awrejcewicz J, Kudra G (2005) Modeling, numerical analysis and application of triple physical pendulum with rigid limiters of motion. Arch Appl Mech 74(11-12):746-753

8. Awrejcewicz J, Kudra G, Wasilewski G (2008) Chaotic zones in triple pendulum dynamics observed experimentally and numerically. Appl Mech Mater 9:1-17

9. Awrejcewicz J, Wasilewski G, Kudra G, Reshmin S (2012) An experiment with swinging up a double pendulum using feedback control. J Comput Syst Sci Int 51(2):176-182 
10. Bendersky S, Sandler B (2006) Investigation of a spatial double pendulum: an engineering approach. Discret Dyn Nat Soc 2006:122

11. Brockett RW, Hongyi L (2003) A light weight rotary double pendulum: maximizing the domain of attraction. 42nd IEEE CDC 4:3299-3304

12. Marsden JE, Scheurle J (1993) Lagrangian reduction and the double spherical pendulum. Z Angew Math Phys 44(1):17-43
13. Lai SK, Lim CW, Lin Z, Zhang W (2011) Analytical analysis for large-amplitude oscillation of a rotational pendulum system. Appl Math Comput 217(13):6115-6124

14. Chaturvedi NA, McClamroch NH, Bernstein DS (2008) Stabilization of a 3D axially symmetric pendulum. Automatica 44(9):22582265 